\title{
Precise Measurement of the Deuteron Elastic Structure Function $A\left(Q^{2}\right)$
}

D. Abbott, ${ }^{5}$ A. Ahmidouch, ${ }^{7,10}$ H. Anklin, ${ }^{8}$ J. Arvieux, ${ }^{11,12}$ J. Ball,, 11 S. Beedoe, ${ }^{10}$ E. J. Beise,,${ }^{3}$ L. Bimbot, ${ }^{12}$ W. Boeglin, ${ }^{8}$ H. Breuer, ${ }^{3}$ R. Carlini, ${ }^{5}$ N. S. Chant, ${ }^{3}$ S. Danagoulian, ${ }^{10}$ K. Dow, ${ }^{7}$ J.-E. Ducret, ${ }^{1}$ J. Dunne, ${ }^{5}$ R. Ent, ${ }^{5}$ L. Ewell, ${ }^{3}$ L. Eyraud, ${ }^{4}$ C. Furget, ${ }^{4}$ M. Garçon, ${ }^{1}$ R. Gilman, ${ }^{5,6}$ C. Glashausser, ${ }^{6}$ P. Gueye,${ }^{5}$ K. Gustafsson, ${ }^{3}$ K. Hafidi, ${ }^{1}$ A. Honegger, ${ }^{2}$ J. Jourdan, ${ }^{2}$ S. Kox,${ }^{4}$ G. Kumbartzki, ${ }^{6}$ L. Lu, ${ }^{4}$ A. Lung, ${ }^{3}$ D. Mack,${ }^{5}$ P. Markowitz, ${ }^{8}$ J. McIntyre, ${ }^{6}$ D. Meekins, ${ }^{5}$ F. Merchez,${ }^{4}$ J. Mitchell,${ }^{5}$ R. Mohring, ${ }^{3}$ S. Mtingwa, ${ }^{10}$ H. Mrktchyan, ${ }^{9}$ D. Pitz,,${ }^{1,3}$ L. Qin, ${ }^{5}$ R. Ransome, ${ }^{6}$ J.-S. Réal, ${ }^{4}$ P. G. Roos,${ }^{3}$ P. Rutt, ${ }^{6}$ R. Sawafta, ${ }^{10}$ S. Stepanyan, ${ }^{9}$ R. Tieulent, ${ }^{4}$ E. Tomasi-Gustafsson,,${ }^{111}$ W. Turchinetz, ${ }^{7}$ K. Vansyoc, ${ }^{5}$ J. Volmer, ${ }^{5}$ E. Voutier ${ }^{4}$ W. Vulcan, ${ }^{5}$ C. Williamson, ${ }^{7}$ S. A. Wood,${ }^{5}$ C. Yan, ${ }^{5}$ J. Zhao, ${ }^{2}$ and W. Zhao ${ }^{7}$

(The Jefferson Lab t ${ }_{20}$ Collaboration)

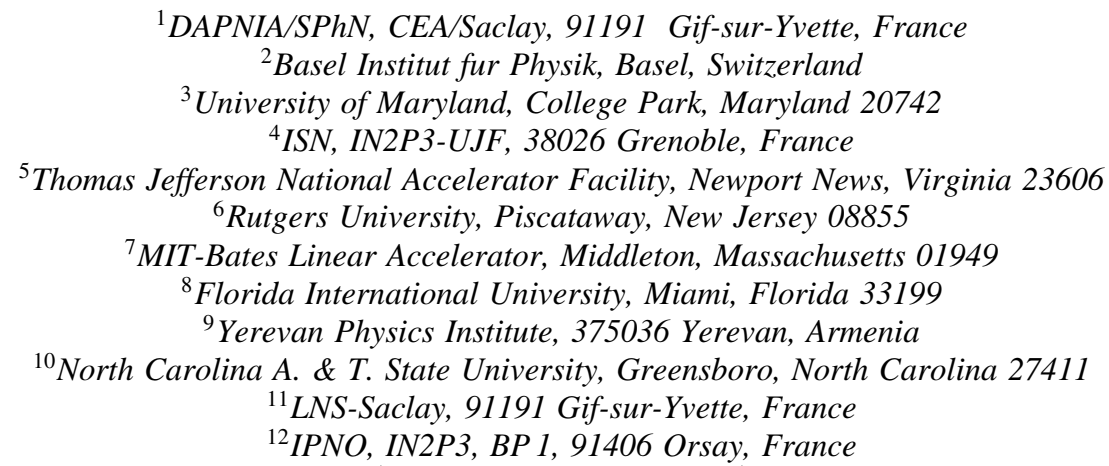

(Received 29 October 1998)

\begin{abstract}
The $A\left(Q^{2}\right)$ structure function in elastic electron-deuteron scattering was measured at six momentum transfers $Q^{2}$ between 0.66 and $1.80(\mathrm{GeV} / c)^{2}$ in Hall C at Jefferson Laboratory. The scattered electrons and recoil deuterons were detected in coincidence, at a fixed deuteron angle of $60.5^{\circ}$. These new precise measurements resolve discrepancies between older sets of data. They put significant constraints on existing models of the deuteron electromagnetic structure, and on the strength of isoscalar meson exchange currents. [S0031-9007(99)08479-3]
\end{abstract}

PACS numbers: 25.30.Bf, 13.40.Gp, 21.45.+v, 27.10.+h

The deuteron is the only two nucleon bound state and, as such, is one of the most fundamental systems in nuclear physics. Measurements of its electromagnetic properties have been invaluable to our understanding of the nucleon-nucleon interaction and the role of meson and isobar degrees of freedom in nuclear systems. At intermediate to high momentum transfer, it remains a challenge to explore the limitations of the mesonucleonic picture of nuclei and unravel the possible role of the quark substructure of nucleons in nuclear structure. The deuteron electromagnetic form factors as measured in elastic $e-d$ scattering provide a crucial test for any model of the deuteron. In this paper, new measurements of the deuteron elastic structure function $A\left(Q^{2}\right)$ are presented in an intermediate momentum transfer region, where previous experiments [1-4] differ by as much as $40 \%$ from each other and where theoretical models have been recently refined.

Assuming single photon exchange, the electrondeuteron unpolarized elastic differential cross section can be written as

$$
\frac{d \sigma}{d \Omega}=\sigma_{\mathrm{NS}}\left[A\left(Q^{2}\right)+B\left(Q^{2}\right) \tan ^{2} \frac{\theta_{e}}{2}\right],
$$

where $\sigma_{\mathrm{NS}}$ is the Mott cross section multiplied by the deuteron recoil factor [5], $Q$ is the four-momentum transfer, and $\theta_{e}$ is the electron scattering angle. $A\left(Q^{2}\right)$ and $B\left(Q^{2}\right)$ are two structure functions [quadratic combinations of the three electromagnetic form factors (charge monopole, quadrupole, and magnetic dipole)] which characterize a spin 1 nucleus:

$$
\begin{gathered}
A\left(Q^{2}\right)=G_{C}^{2}\left(Q^{2}\right)+\frac{8}{9} \tau^{2} G_{Q}^{2}\left(Q^{2}\right)+\frac{2}{3} \tau G_{M}^{2}\left(Q^{2}\right), \\
B\left(Q^{2}\right)=\frac{4}{3} \tau(1+\tau) G_{M}^{2}\left(Q^{2}\right),
\end{gathered}
$$

with $\tau=Q^{2} / 4 M_{d}^{2}$. In the kinematic conditions of the present experiment, $A$ is dominated by the contribution from $G_{Q}$ and, to a lesser extent, by the one from $G_{C}$, which exhibits a node [6,7]. Existing measurements of $B$ [8] indicate that its contribution to our forward angle cross sections is always smaller than $1.6 \%$.

The two photon exchange contribution, where a virtual photon couples to each nucleon, has been estimated to contribute up to a few percent to the $e$ - $d$ cross sections 
[9]. Because of uncertainties coming from approximations in the estimate, this double scattering contribution is neglected here, but deserves more investigation.

The measurements were performed in Hall $\mathrm{C}$ at Jefferson Laboratory, as part of an experiment devoted to the determination of the deuteron tensor polarization [7], with specific conditions for the precise determination of absolute cross sections. An $80 \mu \mathrm{A}$ continuous electron beam was used on a $4.45 \mathrm{~cm}$ thin-walled aluminum cell filled with liquid deuterium. The target cryogen contained $99 \%$ deuterium and $1 \%$ hydrogen. The beam spot was rastered on the target ( $\pm 1 \mathrm{~mm}$ in both directions), and the density reduction due to beam heating was measured to be about $1.3 \%$. The integrated beam charge was measured with three resonant cavity monitors and a parametric current transformer. The beam energies, between 1412 and $4050 \mathrm{MeV}$, were measured to $0.1 \%$. The scattered electrons were detected in the Hall $\mathrm{C}$ high momentum spectrometer (HMS) [10]. This spectrometer consists of three quadrupoles and one dipole, all supraconducting magnets. It is equipped with a detector package of two drift chambers (six planes each), two scintillator hodoscopes (two planes each), and electromagnetic calorimeter, and a gas Čerenkov detector, resulting in an electron detection efficiency of $94-99 \%$.

For a precise definition of the electron solid angle and to minimize the acceptance mismatch with the recoil deuteron detector, the HMS was equipped with a specially designed tungsten collimator $( \pm 8.01 \mathrm{mrad}$ in horizontal, $\pm 43.8 \mathrm{mrad}$ in vertical). The resulting solid angle, as determined from a Monte Carlo simulation, was $1.386 \mathrm{msr}$. The recoil deuterons were detected in coincidence with the electrons using the upstream two scintillators of the POLDER polarimeter [11], after passing through a fixed angle $\left(60.5^{\circ}\right)$ magnetic channel. The deuteron channel (DC) was made of conventional magnets, three quadrupoles and one dipole. The second quadrupole had a large asymmetric aperture and was equipped with additional coils providing a sextupolar field for second order corrections. To first order, the magnetic tune was a point-to-point focus in the vertical plane, and zero magnification and kinematics dependent focusing in the horizontal dispersive plane. Multiple scattering and absorption in the DC were small compared to that in the target fluid. The small acceptance mismatch between the HMS and the DC (1\% to $3.7 \%)$ was modeled at the lowest three kinematic points, and measured at the highest three points. The elastic $e-d$ events were identified unambiguously through the HMS determination of the electron momentum and angle, the $e-d$ coincidence timing peak, and the deuteron energy loss in the POLDER scintillators. Target wall contributions (which were subtracted) were approximately $0.1 \%$.

In the data analysis, a cut at $-4 \%$ was applied to the electron momentum relative to the elastic peak. The corresponding corrections (about 20\%) due to losses in
TABLE I. Measured $e-d$ cross sections and extracted values of $A$, with statistical and systematic errors. Values of $B$ are from a fit to the world data.

\begin{tabular}{lcccccc}
\hline \hline$Q^{2}(\mathrm{GeV} / c)^{2}$ & 0.657 & 0.786 & 1.017 & 1.178 & 1.510 & 1.790 \\
\hline$\theta_{e}(\mathrm{deg})$ & 35.67 & 33.53 & 29.83 & 27.52 & 23.29 & 20.27 \\
$d \sigma / d \Omega(\mathrm{nb} / \mathrm{sr})$ & 77.1 & 43.3 & 19.4 & 11.6 & 5.20 & 2.75 \\
$B \times 10^{8}$ & 5090 & 2030 & 441 & 148 & 22 & 3.6 \\
$A \times 10^{6}$ & 323 & 194 & 88.3 & 51.9 & 20.9 & 9.77 \\
$\Delta A_{\text {stat }} / A(\%)$ & 0.6 & 0.8 & 0.9 & 0.8 & 0.9 & 1.1 \\
$\Delta A_{\text {syst }} / A(\%)$ & 3.7 & 3.8 & 3.9 & 4.0 & 4.8 & 5.5 \\
\hline \hline
\end{tabular}

the radiative tail were calculated according to Ref. [12]. The deuteron losses through nuclear scattering and absorption were estimated from measured and calculated deuteron-nucleus $(d-A)$ total cross sections. Calculations, necessary in the case of $d-d$ scattering where no data are available, were based on the Glauber formalism following Ref. [13]. These losses amounted to $3.2 \%$ to $6.2 \%$, depending on the kinematical setting.

Our results are shown in Table I. The systematic errors on $A\left(Q^{2}\right)$ come from uncertainties in the beam current measurements $(0.5 \%)$, the beam energy (about $1 \%)$, the target length and density (1.3\%), the electron angle $(2.3 \%-4.7 \%)$ and solid angle $(1 \%)$, the electron tracking efficiency $(0.2 \%-1.2 \%)$, the radiative corrections $(1.5 \%)$, the mismatch between DC and HMS (0.5\%$1 \%)$, and the deuteron losses $(0.6 \%-1.2 \%)$. These were combined quadratically. The small $B\left(Q^{2}\right)$ contribution was subtracted using a fit to the world data, with no additional contribution to the systematic errors. Elastic electron-proton $(e-p)$ cross sections were measured for all six energies with the HMS only, and for one energy with the protons in coincidence in the DC. They are, on average, $2 \%$ higher than the cross sections extracted from fits to the $e-p$ data, which is consistent with the precision of these

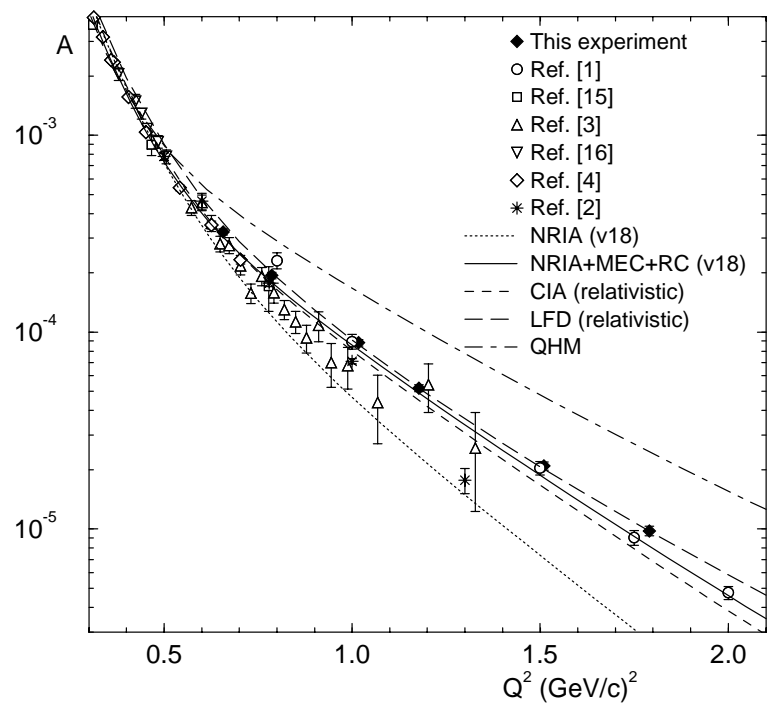

FIG. 1. $A\left(Q^{2}\right)$ : The data are from previous experiments $[1-4,15,16]$ and from this work. The curves are from calculations [20,22-24] discussed in the text. 


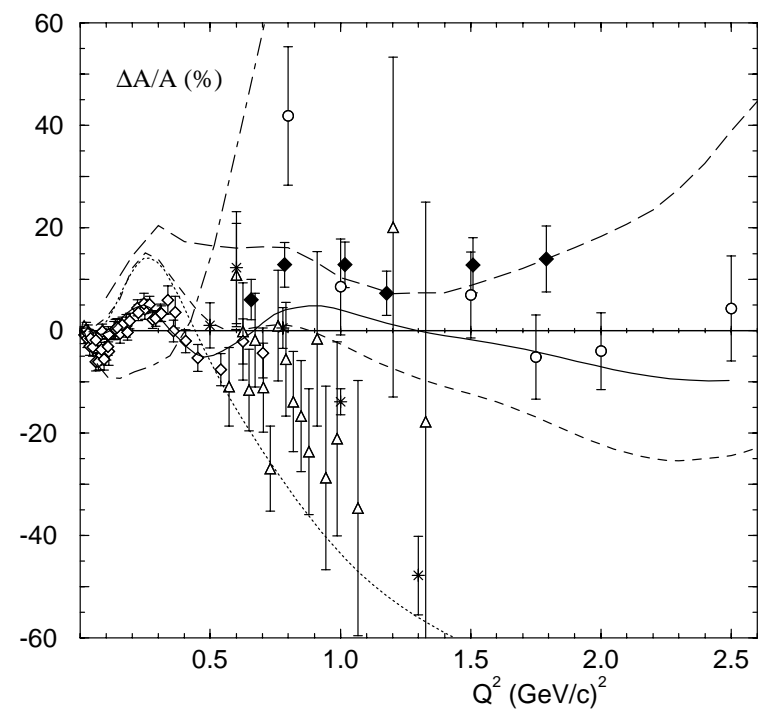

FIG. 2. $A\left(Q^{2}\right)$ deviation (in percent) from a fit to previous data, used as an arbitrary reference. For clarity, only previous data from Refs. [1-4] are shown. See Fig. 1 for experiments and calculation legends. Our experimental error bars combine statistical and systematic errors in quadrature.

data and of this paper. More details on the experiment and data analysis will be found elsewhere [14].

Our results are plotted in Figs. 1 and 2. They smoothly approach the data from ALS [4], but are in clear disagreement with the lowest $Q^{2}$ point from SLAC [1]. Above $1(\mathrm{GeV} / c)^{2}$, our results are in good agreement with the SLAC data (significantly higher than the CEA [3] and Bonn [2] data). The CEA data were measured with background contributions that might not have been subtracted reliably. From Bonn, only the highest $Q^{2}$ point is a determination of $A$ independent of previous measurements. New results from an experiment in the Hall A facility at the Jefferson Laboratory also exist [17].

Several classes of models can be used to calculate the deuteron form factors and $A\left(Q^{2}\right)$. We will give only a few examples based on the most recent calculations. For a review of earlier work, see, e.g., Refs. [4,6]. All calculations are sensitive to the nucleon form factors and, in particular, to the poorly known neutron charge form factor $\left(G_{E}^{n}\right)[4,18,19]$. For instance, a $30 \%$ change in $G_{E}^{n}$ results in about a $6 \%$ change in $A$, in the $Q^{2}$ range of the data presented here. $G_{E}^{n}$ should in the near future, be better determined from several experiments. Different calculations for the deuteron form factors, including the ones discussed below, do not use the same parametrization of the nucleon form factors. The nonrelativistic impulse approximation (NRIA), using recent nucleon-nucleon potentials, underestimates the structure function $A\left(Q^{2}\right)$. The addition of meson exchange currents (MEC) and relativistic corrections (RC) improves the description, as illustrated by a calculation using the Argonne $v_{18}$ potential [20]. There is, however, some uncertainty about the pair term contribution [19] and about the exact strength of the $\rho \pi \gamma$ contribution $[18,21]$, so that the good description given by the full nonrelativistic calculation of Ref. [20] still requires confirmation. The new $A\left(Q^{2}\right)$ data should help fix these isoscalar MEC more accurately. Note that most nucleon-nucleon potentials, whether used in relativistic or nonrelativistic calculations, are adjusted to fit the nucleonnucleon phase shifts up to only about $350 \mathrm{MeV}$, which might not be high enough to match the $Q^{2}$ region of the present measurements. Isobar configurations (mostly $\Delta \Delta$ ) could also play a role in the deuteron electromagnetic structure [19]. For a better description of high $Q^{2}$ data, several fully covariant approaches have been developed. A solution of the Bethe-Salpeter equation using the Gross approximation (CIA) [22] and a calculation using lightfront dynamics (LFD) [23] give similar results and are in reasonable agreement with our data. They are, however, systematically too high at lower $Q^{2}$, and differ at higher $Q^{2}$. The LFD calculation [23] does not include the $\rho \pi \gamma$ MEC. In quark-hadron hybrid models (QHM), the addition of quark exchange terms leads to an overestimate of $A\left(Q^{2}\right)$ [24]. Finally, by parametrizing $A$ with a falloff in $Q^{-2 n}$, the exponent $n$ is found to increase from 2.7 to 4.5 in the region of this experiment, still smaller than the value $n=10$ predicted asymptotically from quark counting rules [25].

In conclusion, we have measured the $A\left(Q^{2}\right)$ deuteron structure function in an intermediate momentum transfer region. Our measurements resolve discrepancies between older experiments and yield significant constraints on the most recent and refined models of the deuteron electromagnetic structure. Their precision also requires a reexamination of small contributions such as two photon exchange. Finally, they were performed at the same kinematics as polarization observables [7] and will be used to extract the deuteron charge monopole and quadrupole form factors with good accuracy.

We acknowledge the outstanding work of the TJNAF accelerator division and the Hall $\mathrm{C}$ engineering staff. We thank the Indiana University Cyclotron Facility for its technical help with some components of the deuteron channel. We also thank R. Lombard and J. Van de Wiele for guidelines concerning the $d-A$ total cross section calculations, as well as M. Rekalo and J. Tjon for discussions concerning the two photon exchange. This work was supported by the French Centre National de la Recherche Scientifique and the Commissariat à l'Energie Atomique, the U.S. Department of Energy and the National Science Foundation, the Swiss National Science Foundation, and the K. C. Wong Foundation.

[1] R. G. Arnold et al., Phys. Rev. Lett. 35, 776 (1975).

[2] R. Cramer et al., Z. Phys. C 29, 513 (1985).

[3] J. E. Elias et al., Phys. Rev. 177, 2075 (1969).

[4] S. Platchkov et al., Nucl. Phys. A510, 740 (1990). 
[5] R. W. McAllister and R. Hofstadter, Phys. Rev. 102, 851 (1956).

[6] M. Garçon et al., Phys. Rev. C 49, 2516 (1994).

[7] C. Furget et al., Acta Phys. Pol. B 29, 3301 (1998).

[8] P.E. Bosted et al., Phys. Rev. C 42, 38 (1990), and references therein.

[9] F. M. Lev, Sov. J. Nucl. Phys. 21, 45 (1975), and references therein.

[10] C. Yan et al., Report No. CEBAF-R-92-002, 1992.

[11] S. Kox et al., Nucl. Instrum. Methods Phys. Res., Sect. A 346, 527 (1994); L. Eyraud, Doctorate thesis, UJFGrenoble, 1998; Report No. ISN-98-101.

[12] L. W. Mo and Y. S. Tsai, Rev. Mod. Phys. 41, 205 (1969); Y.S. Tsai, Report No. SLAC-PUB-848, 1971.

[13] S. K. Charagi and S. K. Gupta, Phys. Rev. C 41, 1610 (1990).

[14] A. Honneger, Doctorate thesis, Basel University (unpublished); D. Pitz, Doctorate thesis, University of Caen (unpublished)
[15] C. D. Buchanan and R. Yearian, Phys. Rev. Lett. 15, 303 (1965).

[16] S. Galster et al., Nucl. Phys. B32, 221 (1971).

[17] L.C. Alexa et al., The Jefferson Lab Hall A Collaboration, preceding Letter, Phys. Rev. Lett. 82, 1374 (1999).

[18] P. Sarriguren et al., Phys. Lett. B 228, 285 (1989).

[19] A. Amghar et al., Eur. Phys. J. A 1, 85 (1998).

[20] R. B. Wiringa et al., Phys. Rev. C 51, 38 (1995).

[21] B. Mosconi and P. Ricci, Few-Body Syst. 8, 159 (1990).

[22] J. W. Van Orden et al., Phys. Rev. Lett. 75, 4369 (1995).

[23] J. Carbonell et al., Phys. Rep. 300, 215 (1998).

[24] A. Buchmann et al., Nucl. Phys. A496, 621 (1989).

[25] S. J. Brodsky and G. R. Farrar, Phys. Rev. Lett. 31, 1153 (1973); V. Matveev et al., Nuovo Cimento Lett. 7, 719 (1973). 